Submitted for publication in the ECOSS Proceedings, 9-13 Sept. 1996, Genova, Surface Science

\title{
Study of CO Oxidation over Ru (0001) at High Gas Pressures
}

\author{
C. Stampfl and M. Scheffler \\ Fritz-Haber-Institut der Max-Planck-Gesellschaft, Faradayweg 4-6, D-14 195 Berlin-Dahlem, \\ Germany
}

(October 17, 2018)

Experiments performed at high gas partial pressures have demonstrated that the kinetics of the $\mathrm{CO}$ oxidation reaction at $\mathrm{Ru}(0001)$ is different and somewhat anomalous compared to that over other transition metal surfaces and, in particular, the turnover rate is exceptionally high. In order to gain insight into the underlying reasons for this behavior, we performed density functional theory calculations using the generalized gradient approximation for the exchange-correlation functional. We find that the high rate is due to a weakly, but nevertheless well bound, $(1 \times 1)$ oxygen adsorbate layer which may form for high $\mathrm{O}_{2}$ pressures but not under usual ultra high vacuum conditions. The calculations indicate that reaction to $\mathrm{CO}_{2}$ occurs both via scattering of gas-phase CO molecules as well as by CO molecules weakly adsorbed at vacancies in the oxygen adlayer, where the latter mechanism dominates the rate.

\section{INTRODUCTION}

The oxidation of carbon monoxide at transition metal surfaces is one of the most extensively studied and best understood heterogeneous catalytic reactions (see, for example Refs. [1] and references therein). Nevertheless, very little in fact, is actually known about the reaction pathway on a microscopic level. Recently there has been a resurge of interest in such surface chemical reactions which has been stimulated by advances in surface science techniques (e.g. molecular and atomic beam experiments [2] and high pressure catalytic reactors [3]). These techniques have enabled new information to be obtained concerning the behavior of chemisorbed reaction partners. Recent studies using high gas pressure catalytic 
reactors (e.g. operating at $\sim 10$ torr with $\mathrm{CO} / \mathrm{O}_{2}$ pressure ratios $<1$ ) have reported unusual behavior for the $\mathrm{CO}$ oxidation reaction over $\mathrm{Ru}(0001)$ [4]: $(i)$ The rate of $\mathrm{CO}_{2}$ production is significantly higher than at other transition metal surfaces; in contrast, under ultra high vacuum (UHV) conditions, $\mathrm{Ru}(0001)$ is notably the poorest catalyst for this reaction [1]. (ii) The measured kinetic data (e.g., activation energy, and temperature and pressure dependencies of the rate) is markedly different to that of other substrates. (iii) As opposed to other transition metal catalysts ( $\mathrm{Pt}, \mathrm{Pd}, \mathrm{Ir}$, and $\mathrm{Rh}$ ), highest rates of $\mathrm{CO}_{2}$ formation occur for high surface oxygen concentrations (one monolayer was proposed on the basis of auger electron spectroscopy measurements). (iv) Almost no chemisorbed CO was detected during or after the reaction. On the basis of these findings, it was speculated that an Eley-Rideal (E-R) mechanism is operational as opposed to the "usual" Langmuir-Hinshelwood (L-H) mechanism. In the E-R mechanism, the reaction occurs between gas-phase and chemisorbed particles, while in the L-H mechanism, the reaction occurs between species both chemisorbed on and in thermal equilibrium with the surface. So far E-R mechanisms have only recently been experimentally confirmed, and in most cases they are for somewhat artificial reactions between gas-phase atomic hydrogen or deuterium (from a beam source) with chemisorbed atoms [5].

In the present paper we describe results our theoretical study aimed at understanding the experimental findings described above for the oxidation of $\mathrm{CO}$ at $\mathrm{Ru}(0001)$.

\section{CALCULATION METHOD}

We performed density functional theory (DFT) calculations using the pseudopotential

plane wave method and the supercell approach [6]. Surfaces were modelled by unit cells of $(2 \times 2)$ and $(1 \times 1)$ periodicity with four layers of $\mathrm{Ru}(0001)$ and a vacuum region equivalent to thirteen such layers. The ab initio fully separable pseudopotentials were created using the scheme of Troullier and Martins [7]. Plane wave cut-offs of 40 Ry, with three k-points [8] in the surface Brillouin zone of the $(2 \times 2)$ cell were used, as well as a 60 Ry cut-off with fifty-two 
k-points in the surface Brillouin zone of the $(1 \times 1)$ cell. Results of calculations performed using these different basis sets indicated that the smaller basis set provides a sufficiently accurate description for the present investigation [9]. The generalized gradient approximation (GGA) of Perdew et al. [10] was employed for the exchange-correlation functional and it was used in creating the pseudopotential as well as in the total-energy functional. It is therefore treated in a consistent way. The position of all atoms were allowed to relax except the bottom two Ru layers which were held at their bulk positions. We like to point out that the present study represents the first investigation of a surface chemical reaction (to be distinguished from dissociative chemisorption) using such theoretical methods. Further details about the calculations can be found in Refs. [9, 11, 12].

\section{ADSORPTION OF O ON RU (0001)}

Given that the $\mathrm{CO}$ oxidation experiments identified that highest rates of $\mathrm{CO}_{2}$ production occurred for high $\mathrm{O}_{2}$ partial pressures and concomitantly high oxygen coverages, an important part of the theoretical study is therefore to investigate the structure and stability of high coverage $\mathrm{O}$ adlayers on $\mathrm{Ru}(0001)$. It is known that using $\mathrm{O}_{2}$, under $\mathrm{UHV}$ conditions

at $300 \mathrm{~K}$, the saturation coverage is close to $\Theta=0.5$. Earlier we reported the results of DFT-GGA calculations for various adsorbate phases of oxygen on $\mathrm{Ru}(0001)$ and found that an even higher coverage should be stable on the surface [9]. In particular, a structure with a $(1 \times 1)$ periodicity and coverage $\Theta=1$ where the oxygen atoms occupy the hcp-hollow sites. (The coverage $\Theta$ is defined as the ratio of the concentration of adparticles to that of substrate atoms in the topmost layer.) This result indicated that the formation of the $\Theta=1$ structure under UHV conditions from gas phase $\mathrm{O}_{2}$ is kinetically hindered, but that by offering atomic oxygen, this phase should be attainable. Indeed this structure was successfully created by starting from the $\Theta=1 / 2$ phase and offering additional atomic oxygen to the surface via the dissociation of $\mathrm{NO}_{2}$ [12]. The determined surface atomic structure of the $(1 \times 1)$ phase by the low-energy electron diffraction (LEED) intensity analysis of Ref. [12] 
agreed very well with that predicted by the DFT-GGA calculations. The calculations show that the adsorption energy of oxygen in this phase is significantly weaker than it is in the lower coverage ordered phases $($ of $(2 \times 2)$ and $(2 \times 1)$ periodicities). This can be seen from Fig. 1 which shows the adsorption energy, with respect to $\mathrm{O}_{2}$ gas, for oxygen in the hcpand fcc-hollow sites at various coverages. The region over which dissociative chemisorption of oxygen is kinetically hindered is indicated as the shaded region. It can be seen that the hcp-hollow site is favored at all coverages, where the adsorption energy of the $(2 \times 2)$ phase is the greatest. This suggests that at low coverages island formation will occur with a $(2 \times 2)$ periodicity which is consistent with experimental observations [13,11]. With subsequently increasing coverage, the adsorption energy decreases which reflects a repulsive adsorbateadsorbate interaction, and indicates that no island formation will take place in the coverage regime $\Theta=0.25$ to 1 . Furthermore, with increasing coverage from $\Theta=0.25$, the difference in adsorption energy between the fcc- and hcp-hollow sites decreases, and at coverage one the difference is very small $(\sim 0.06 \mathrm{eV})$.

Catalysis involving high $\mathrm{O}_{2}$ pressures is therefore likely to involve oxygen coverages that approach one monolayer on the surface because the impingement rate is proportional to the partial pressure which means that there is a significantly higher attempt frequency to overcome activation barriers for dissociative adsorption than under UHV conditions. We therefore initially assumed in our investigation of the oxidation of $\mathrm{CO}$ at $\mathrm{Ru}(0001)$ that the $(1 \times 1)$ phase covers the surface.

\section{OXIDATION OF CO AT $(1 \times 1)-O / R U(0001)$}

For the catalytic oxidation of $\mathrm{CO}$, it is necessary that a $\mathrm{CO}$ molecule reacts with an oxygen atom to yield $\mathrm{CO}_{2}$. From the experiments it had been speculated that $\mathrm{CO}$ may achieve this from the gas-phase without adsorbing on the surface first, i.e. the E-R mechanism. To investigate the possibility of this scenario, calculations were first carried out to determine

whether $\mathrm{CO}$ could adsorb on the $(1 \times 1)$ oxygen covered $\mathrm{Ru}(0001)$ surface. The sites con- 
sidered were the on-top and fcc-hollow sites, with respect to the $\mathrm{Ru}(0001)$ substrate, and a bridge site between two adsorbed $\mathrm{O}$ atoms. It was found that at all these sites, $\mathrm{CO}$ is unstable, thus preventing the L-H mechanism on the perfectly ordered $(1 \times 1)-\mathrm{O} / \mathrm{Ru}(0001)$ surface.

To investigate the possibility of reaction via an E-R mechanism, we evaluate an appropriate cut through the high-dimensional potential energy surface (PES); this cut is defined by two variables: the vertical position of the $\mathrm{C}$ atom and the vertical position of the $\mathrm{O}$ adatom below the molecule. In order of ease of analysis, the $\mathrm{CO}$ axis is held perpendicular to the surface. The resulting PES is presented in Fig. 2 where the coordinate system is shown as the inset. The most favorable pathway can quickly be seen: it has an energy barrier of about $1.6 \mathrm{eV}$ and formation of $\mathrm{CO}_{2}$ is achieved via an upward movement of the $\mathrm{O}$ adatom by $\approx 0.4$ $\AA$ towards the $\mathrm{CO}$ molecule. Due to the similar masses of $\mathrm{O}$ and $\mathrm{C}$, it is likely that the impinging CO molecule will impart a significant amount of energy to the O adatom, thus stimulating its vibrations and facilitating its motion (indicated by the oscillations in the dot-dashed line). This would then bring the system to the transition state of the reaction (marked by the asterisk). The newly formed $\mathrm{CO}_{2}$ molecule is strongly repelled from the surface and moves into the gas phase region with a large energy gain of $1.95 \mathrm{eV}$.

As mentioned, the PES of Fig. 2 corresponds to a constrained situation of the surface-CO angle. When this constraint is dropped, i.e., when the tilt angle of the CO axis is allowed to relax, we find that the energy barrier is reduced to $1.1 \mathrm{eV}$, and also that the position of the saddle point occurs closer to the surface (by $0.3 \AA$ ). The geometry of the identified transition state at the saddle point is depicted in Fig. 3. The optimum tilt angle with respect to the surface normal is $49^{\circ}$ which corresponds to a "bond angle" of $131^{\circ}$ for the " $\mathrm{CO}_{2}$-like" complex. It is interesting to note that this geometry is similar to that associated with the $\mathrm{CO}_{2}^{-}$ion [15] and to that proposed for the "activated complex" for the CO oxidation reaction over other transition metal surfaces [16] in which the reaction proceeds via the L-H mechanism.

In Fig. 4 the energy diagram for the proposed reaction pathway for the E-R mechanism 
is given. Interestingly, the calculations show that there is a physisorption well for $\mathrm{CO}$ (over all of the surface unit cell), as well as for $\mathrm{CO}_{2}$, above the surface. The calculated depths however $(\sim 0.04 \mathrm{eV})$, are likely to be underestimated because the employed exchangecorrelation functional does not describe the long-range van der Waals type interactions. It can be seen that with respect to the free $\mathrm{CO}$ and $\frac{1}{2} \mathrm{O}_{2}$ there is an energy gain of $1.20 \mathrm{eV}$ on adsorption of oxygen into a vacancy in the $(1 \times 1)$ structure, an energy barrier for reaction to $\mathrm{CO}_{2}$, and a significant energy gain on formation of $\mathrm{CO}_{2}$ which leaves an oxygen vacancy behind on the surface. This predicted mechanism and associated energetics could possibly be tested by molecular beam experiments. It is, however, unlikely that this process alone can explain the high rate measured in the high pressure catalytic reactor experiments given the height of the activation energy barrier, which is high for an E-R mechanism.

It is conceivable that $\mathrm{CO}$ molecules adsorb at vacancies in the O-adlayer, i.e., at sites at which an oxygen atom has been removed (e.g. by the above described E-R reaction). We find that $\mathrm{CO}$ can weakly adsorb in such a vacancy where the adsorption energy is $0.85 \mathrm{eV}$ - significantly less than on the clean surface which we calculate to be $1.68 \mathrm{eV}$ (the experimental value has been determined to be $1.66 \mathrm{eV}[18]$ ). Adsorption of $\frac{1}{2} \mathrm{O}_{2}$ in such a vacancy is more favorable, with an adsorption energy of $1.20 \mathrm{eV}$. Using these adsorption energies of $\mathrm{CO}$ and $\frac{1}{2} \mathrm{O}_{2}$, and assuming thermal equilibrium of the $\mathrm{CO}+\mathrm{O}_{2}$ gas and a mixed $\mathrm{CO}+\mathrm{O}$ adlayer, the law of mass action indicates that about $0.03 \%$ of the sites of the $(1 \times 1)$ adlayer will be occupied by $\mathrm{CO}$. (We assumed that the $\mathrm{O}_{2}$ and $\mathrm{CO}$ partial pressures are equal and the temperature is $T=500 \mathrm{~K}$.) In reality the $\mathrm{CO}$ concentration will be even higher because we find that $\mathrm{CO}$ adsorption into an existing $\mathrm{O}$ vacancy can proceed basically without hindrance while $\mathrm{O}$ adsorption $\left(\right.$ from $\mathrm{O}_{2}$ ) is hindered by an energy barrier. Therefore the actual percentage of surface sites occupied by CO will be somewhat higher. A L-H reaction between the adsorbed $\mathrm{CO}$ molecule in a vacancy and a neighboring $\mathrm{O}$ adatom is expected to be particularly efficient and to give rise to the high rate due to the weaker bond strengths of both the adsorbed $\mathrm{CO}$ molecule and $\mathrm{O}$ adatom, as well as their close proximity to one another. In contrast, under UHV conditions, where the high coverage 
phase is unable to form due to kinetic hindrance for $\mathrm{O}_{2}$ dissociation, $\mathrm{Ru}(0001)$ is the poorest catalyst for this reaction. The reason for this is likely to be related to the fact that under these conditions ruthenium binds oxygen (and carbon monoxide) particularly strongly. 


\section{REFERENCES}

[1] T. Engel and G. Ertl, Adv. Catal. 28 (1979) 1; The chemical physics of solid surfaces and heterogeneous catalysis, eds. D. A. King and D. P. Woodruff, Vol. 4, Elsevier (1982).

[2] K. D. Rendulic and A. Winkler, Surf. Sci. 299/300 (1994) 261.

[3] D. W. Goodman, Surf. Sci. 299/300 (1994) 837.

[4] C. H. F. Peden, in Surface Science of Catalysis: In situ probes and reaction kinetics, Eds. D. J. Dwyer and F. M. Hoffmann, Am. Chem. Soc., Washington DC (1992).

[5] E. W. Kuipers, A. Vardi, A. Danon, and A. Amirav, Phys. Rev. Lett. 66 (1991) 116; C. T. Rettner, Phys. Rev. Lett. 69 (1992) 383; C. T. Rettner and D. J. Auerbach, Phys. Rev. Lett. 74 (1995) 4551; C. T. Rettner, J. Chem. Phys. 101 (1994) 1529.

[6] R. Stumpf and M. Scheffler, Comp. Phys. Commun. 79 (1994) 447.

[7] N. Troullier and J. L. Martins, Phys. Rev. B 43 (1991) 1993.

[8] S. L. Cunningham, Phys. Rev. B 10 (1974) 4988.

[9] C. Stampfl and M. Scheffler, Phys. Rev. B, 54, 2868 (1996).

[10] J. P. Perdew, J. A. Chevary, S. H. Vosko, K. A. Jackson, M. R. Pederson, D. J. Singh, and C. Fiolhais, Phys. Rev. B 46 (1992) 6671.

[11] C. Stampfl, Surf. Rev. Lett., in press.

[12] C. Stampfl, S. Schwegmann, H. Over, M. Scheffler, and G. Ertl, Phys. Rev. Let., in press.

[13] K. L. Kostov, H. Rauscher, and D. Menzel, Surf. Sci. 278 (1992) 62.

[14] J. Wintterlin, J. Trost, S. Renisch, R. Schuster, and G. Ertl, to be published.

[15] J. Pacansky, U. Wahlgren, and P. S. Bagus, J. Chem. Phys. 62 (1975) 2740. 
[16] G. W. Coulston and G. L. Haller, J. Chem. Phys. 95 (1991) 6932, and references therein.

[17] W. J. Mitchell and W. H. Weinberg, J. Chem. Phys. 104 (1996) 9127.

[18] H. Pfnür, P. Feulner, H. A. Engelhardt, and D. Menzel, Chem. Phys. Lett. 59 (1978) 481. 


\section{FIGURES}

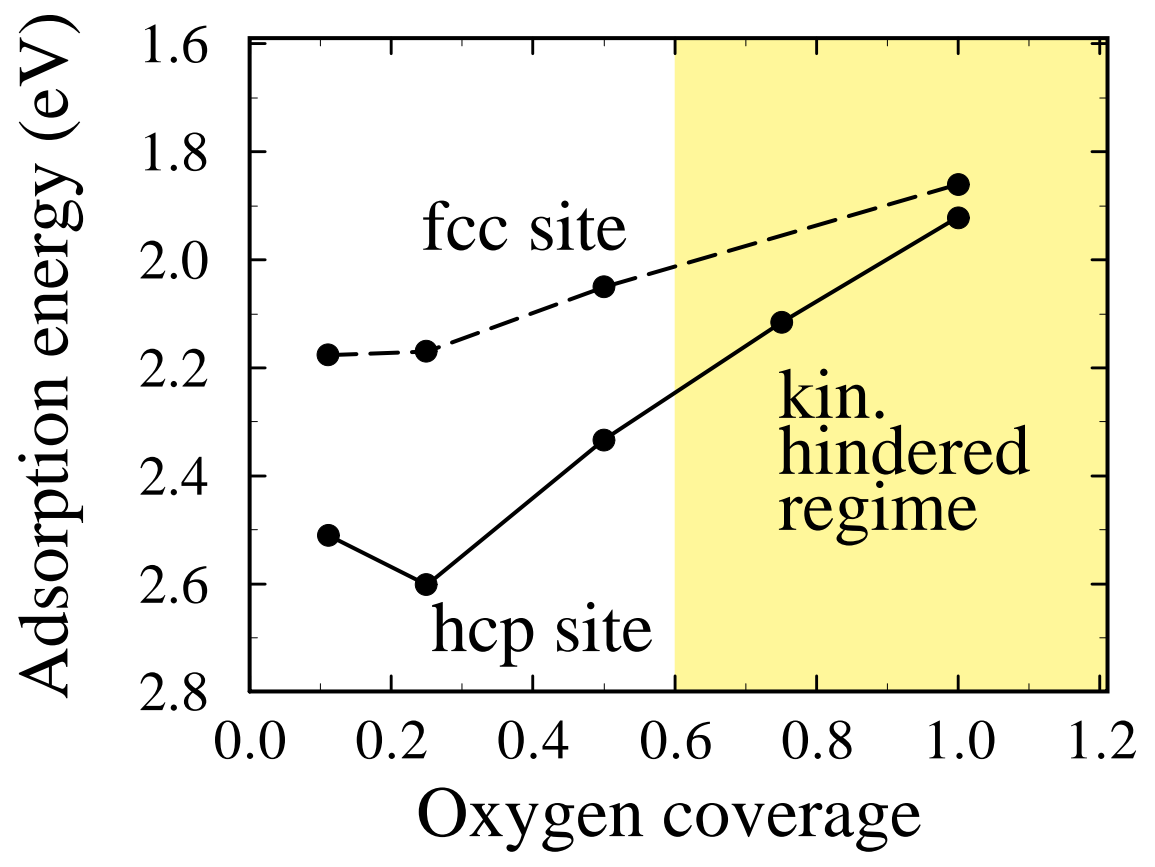

FIG. 1. Adsorption energy of $\mathrm{O}$ on $\mathrm{Ru}(0001)$ with respect to $\frac{1}{2} \mathrm{O}_{2}$ at various coverages for oxygen in the fcc- (dashed line) and hcp-hollow sites (continuous line). 


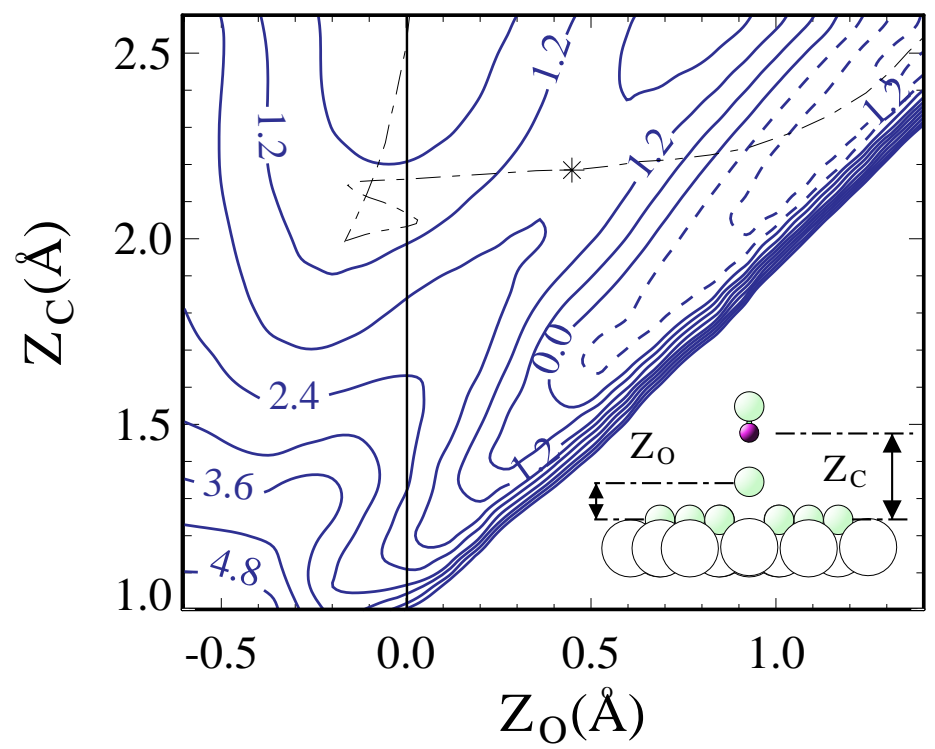

FIG. 2. Potential energy surface (PES) as a function of the positions of the $\mathrm{C}$ atom, $Z_{\mathrm{C}}$, and of the $\mathrm{O}$ adatom, $Z_{\mathrm{O}}$. Positive energies are shown as dashed lines, negative ones as full lines. The contour line spacing is $0.6 \mathrm{eV}$. 


\section{Transition State}

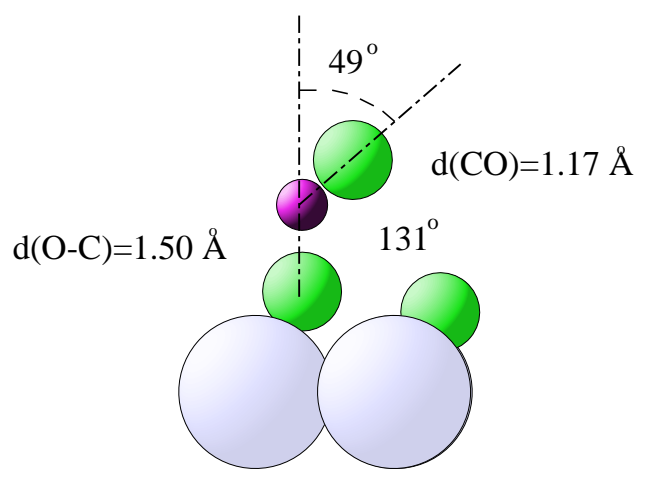

FIG. 3. Illustration of the transition state identified for the reaction of gas-phase CO with adsorbed oxygen when tilting of the $\mathrm{CO}$ axis is considered. 
FIG. 4. Predicted energy diagram for the E-R mechanism of $\mathrm{CO}$ oxidation at $\mathrm{Ru}(0001)$. Note that the depths of the physisorption wells are exaggerated for visibility. 
This figure "fig4.gif" is available in "gif" format from: http://arxiv.org/ps/cond-mat/9611217v1 